\title{
SOME DECOMPOSITIONS OF THE SPACE OF SYMMETRIC TENSORS ON A RIEMANNIAN MANIFOLD
}

\author{
M. BERGER \& D. EBIN
}

\section{Introduction}

In this article we consider a compact $C^{\infty}$ manifold $M$ and endow it with a riemannian structure $g$. For such a riemannian manifold $(M, g)$, the space $A^{p}$ of exterior differential forms carries an elliptic operator and the de Rham laplacian $\Delta$, and has an orthogonal decomposition

$$
A^{p}=\operatorname{ker} \Delta \oplus d A^{p-1} \oplus \delta A^{p+1},
$$

orthogonal with respect to the global scalar product on $A^{p}$. Moreover the dimension of $\operatorname{ker} \Delta$ is equal to the $p$-th Betti number of $M$, thanks to de Rham's theorem.

The simplest space of tensors to consider, besides the $A^{p}$ 's, is the space $S^{2}$ of symmetric bilinear differential forms on $M$. It is natural to look for a decomposition of $S^{2}$ like $(1,1)$. In this article we give all the reasonable decompositions of $S^{2}$, which we are aware of. Unhappily we have no essential applications of them, because of the lack of some kind of a de Rham theorem, connecting topological invariants of $M$ with the dimension of the kernel of the elliptic operators considered on $S^{2}$. However we think it is worthwhile to list and prove these decompositions, hoping the reader will find interest in the problems and questions which naturally arise.

After fixing notations in $\S 2$ we give in $\S \S 3$ and 4 the decomposition (3.1), which essentially yields the subspace $\delta^{-1}(0)$ of $S^{2}$ as the tangent space at $g$ of the space $\mathscr{M} / \mathscr{D}$ of classes of riemannian structures on $M$ under diffeomorphisms. In $\S 5$ we give two decompositions for manifolds of constant scalar curvature which are naturally associated to deformations of the scalar curvature. One of these is due to L. Nirenberg. $\S 6$ lists four elliptic operators on $S^{2}$; in $\S 6 . c$ an application is made to minimal surfaces; Corollary 6.2 was suggested to us by J. Simons. $\S \S 7$ and 8 are concerned with Einstein manifolds. As an application of the decomposition (3.1) of $\S 3$, we find that the space of Einstein

\footnotetext{
Communicated by L. Nirenberg, December 5, 1968. The work of the first author was done under NSF grant GP-8623, and that of the second author was supported by NSF post-doctoral fellowship.
} 
structures is locally finite-dimensional and we also get a result of nondeformability of some Einstein manifolds (Corollary 7.3 and Lemma 7.4).

\section{Notations}

On our fixed compact manifold $M$, we denote by $A^{p}(p=0,1, \ldots, \operatorname{dim}$ $M=n$ ) the $p$-th exterior power $\stackrel{p}{\wedge} T^{*}(M)$ of the cotangent bundle $T^{*}(M)$ of $M$. Hence $C^{\infty}\left(A^{p}\right)$, the space of all $C^{(1)}$-sections of $A^{n}$, is the space of exterior differential forms of degree $p$ (in particular $A^{0}$ is the space of $C^{\circ}$-functions on $M)$. When no confusion is possible, we write $A^{\prime \prime}$ for $C^{\omega \prime}\left(A^{p}\right)$. Similarly $T_{s}^{r}$ represents the space of all tensors on $M$, which are $r$-times covariant and $s$-times contravariant, i.e., $T_{s}^{r}=\left[{ }^{r} T(M)\right] \otimes\left[\otimes^{*} T^{*}(M)\right]$. By $S^{2} \subset T_{2}^{0}$ we understand the bundle (or also $C^{\infty}\left(S^{2}\right)$ ) of symmetric bilinear differential forms.

We endow $M$ with a fixed riemannian structure $g$, making it a ricmannian manifold $(M, g)$. On tensor spaces on $M$ we have the canonical scalar product (point-wise) $(\cdot \mid \cdot)$ and on their sections the global scalar product $\langle\cdot, \cdot\rangle$ $=\int_{M}(\cdot \mid \cdot) v_{g}$, where $v_{g}$ is the canonical measure of $(M, g)$. If $P$ is a differential operator between some tensor bundles over $M$, its formal adjoint $P^{*}$ is uniquely defined by $\langle P \cdot, \cdot\rangle=\left\langle\cdot, P^{*} \cdot\right\rangle$.

Examples. The covariant derivative $\Gamma: T_{s}^{r} \rightarrow T_{s: 1}^{r}$, whose formal adjoint $\nabla^{*}$ will be also denoted by $\delta=\Gamma^{*}: T_{s ! 1}^{\dot{r}} \rightarrow T_{s}^{r}$. In local coordinates:

$$
(\delta T)_{i_{1} \cdots i_{s}}^{j_{1} \cdots i_{r}}=-\sum_{l} \Gamma^{\prime} T_{i_{i_{1}} \cdots i_{s}}^{j_{1} \cdots i_{r}} .
$$

For the restriction of $\delta$ to $S^{2}: \delta: S^{2} \rightarrow A^{1}$, still denoted by $\delta$, the adjoint $\delta^{*}$ is given by the formula:

$$
\left(\delta^{*} \xi\right)(x, y)=\frac{1}{2}\left[\Gamma_{. r} \xi(y)+\Gamma_{, !} \xi(x)\right]=\frac{1}{2} \mathscr{L}^{\prime}{ }^{\prime} g,
$$

where $\mathscr{L}$ is the Lie derivative and $\xi^{\prime}$ the vector field dual (by $g$ ) to the 1 -form $\xi$.

On $S^{2}$ we have the trace: $\operatorname{tr}: S^{2} \rightarrow A^{0}$ (with respect to $g$ understood), since $g$ defines canonically a map $S^{2} \rightarrow T_{1}^{1}$ and $T_{1}^{1}$ is made up of endomorphisms (which have a cononical trace). We will set

$$
T Z=\left\{h \in S^{2}: \operatorname{tr} h=0\right\}=\operatorname{tr}^{-1}(0) \subset S^{\prime 2} .
$$

Example. On $A^{0}$, we have the double covariant derivative, called the hessian: Hess $=\nabla \circ \nabla: A^{0} \rightarrow S^{2}$. Its trace is nothing but -1 , the usual LaplaceBeltrami operator on $A^{0}: \Delta=-\operatorname{tr} \circ$ Hess: $A^{0} \rightarrow A^{0}$ (in local coordinates $\Delta f$ $\left.=-\sum_{l} \nabla^{\prime} \nabla_{l} f=-\sum_{l} \nabla^{l} d_{l} f\right)$. 
Finally, for our riemannian manifold $(M, g)$, we denote by $R$ the curvature tensor, $\rho$ the Ricci curvature $\left(\rho \in S^{2}\right)$ and $\tau$ the scalar curvature $\left(\tau \in A^{0}\right)$. The signs we are choosing are such that, for the standard sphere $\left(S^{n}, g_{0}\right)$ :

$$
\begin{array}{ll}
R(x, y ; x, y)=+1 & \text { for any orthonormal } x, y, \\
\rho(x, x)=n-1 & \text { for any unit vector, } \\
\tau=n(n-1) . &
\end{array}
$$

\section{The first splitting of $S^{2}$}

As is well known, the set $\mathscr{M}$ of riemannian metrics on $M$ is an open (in $C$ topology) convex positive cone in the linear space $C^{\infty}\left(S^{2}\right)$. One approach to a study of riemannian geometry is to consider certain distinguished subsets of $\mathscr{M}$; e.g., those metrics which make $M$ a space form, Einstein manifold, homogeneous space, etc.

The diffeomorphism group $\mathscr{D}$ of $M$ acts on $\mathscr{M}$ in a natural way by pull back. (The usual action on the sections of any tensor bundle.) We denote a metric $g$ acted on by $\eta$ as $\eta^{*}(g)$, and it is easy to see that if $(M, g)$ is a space form, Einstein manifold or homogeneous space, then so is $\left(M, \eta^{*}(g)\right)$. This means that each of our distinguished subsets of $\mathscr{M}$ is a union of orbits of $\mathscr{D}$ in $\mathscr{M}$. Hence each one can also be looked at as a subset of the space of orbits $\mathscr{M} / \mathscr{D}$.

In [4], Ebin has analyzed the action of $\mathscr{D}$ on $\mathscr{M}$ and proved the following:

Fix $g \in \mathscr{M}$ and let $0_{g}$ be the orbit of $\mathscr{D}$ containing $g$. Then there exist a neighborhood $U$ of $g$ in $0_{g}$ and a map $\chi: U \rightarrow \mathscr{D}$ such that if $\eta^{*}(g) \in U$, $\left(\chi\left(\eta^{*}(g)\right)\right)^{*}(g)=\eta^{*}(g)$; i.e., $\chi$ is a local section of the map of $\mathscr{D}$ onto its orbit. Also there is a submanifold $S$ of $\mathscr{M}$ containing $g$ such that the map $F: U \times S$ $\rightarrow \mathscr{M}$ defined by $F(u, s)=(\chi(u))^{*}(s)$ is a diffeomorphism of $U \times S$ onto a neighborhood of $g$ in $\mathscr{M} .{ }^{1}$ Furthermore the tangent space of $S$ at $g$ is the kernel of the operator $\delta: S^{2} \rightarrow T^{*}$.

The existence of $S$ and $F$ is helpful to the study of the local nature of the various distinguished subsets of $\mathscr{M}$. Let $\mathscr{E}$ be such a subset, and $g(t)$ a curve in $\mathscr{M}$, which is contained in $\mathscr{E}$ and such that $g(0)=g$. If $\pi_{2}: U \times S \rightarrow S$ is the projection on the second factor, then

$$
\pi_{2} \circ F^{-1}(g(t)) \text { is a curve in } \mathscr{M}
$$

(defined for all $t$ such that $g(t) \in F(U \times S)$ ). But $h(t)=\pi_{2} \circ F^{-1}(g(t))$ and $g(t)$ are in the same orbit of $\mathscr{D}$ because if $F^{-1}(g(t))=(u, s)$, then $h(t)=s$ and $g(t)=(\chi(u))^{*}(s)$. Hence $h(t)$ is also a curve in $\mathscr{E}$, so $h(t) \subseteq S \cap \mathscr{E}$. Thus any

\footnotetext{
$1 \mathscr{H}, \mathscr{D}$, and $0_{g}$ are all locally Frechét spaces. Therefore we must understand the words "submanifold" and "diffeomorphism" in the sense of ILH-submanifold and diffeomorphism (see [7]).
} 
deformation $g(t)$ of $g$ in the set $\mathscr{E}$ gives rise to a deformation $h(t)$ in $\mathscr{E} \cap S$, such that if $\pi: \mathscr{M} \rightarrow \mathscr{M} / \mathscr{D}$ is the natural projection, $\pi g(t)=\pi h(t)$. Since the tangent space of $S$ at $g$ is $\delta^{-1}(0),\left.\frac{d}{d t} h(t)\right|_{0} \in \delta^{-1}(0)$. Hence to study deformations in $\mathscr{M} / \mathscr{D}$ we need only study curves in $\mathscr{U}$ whose tangent at $g$ is in $\delta^{-1}(0)$. Here we shall prove not the existence of $S$ and $F$ but an infinitesimal version of their construction, that is, we shall show that

$$
C^{\infty}\left(S^{2}\right)=\delta^{-1}(0) \oplus \delta^{*}\left(C^{\cdots}\left(A^{1}\right)\right),
$$

and $\delta^{*}\left(C^{\lrcorner}\left(A^{1}\right)\right)$ is the tangent space of $0_{\imath}($ or $U)$ at $g$.

First we give a plausibility argument that $\delta^{*}\left(C^{\omega}\left(A^{1}\right)\right)$ is the tangent space of $0_{g}$ at $g$ (for details see [4]). We let $\phi_{g}: \mathscr{D} \rightarrow . \| /$ by $\eta \rightarrow \eta^{*}(g)$, and shall show that at the identity of $\mathscr{D}, 2 \delta^{*}: C^{\prime \prime}\left(A^{1}\right) \rightarrow C^{\prime}\left(S^{\prime}\right)$ is the tangent map of $\phi_{\prime \prime \prime}$.

If $\eta(t)$ is a curve in $\mathscr{D}$, with $\eta(0)=I d$, then for each $p \in M,\left.\frac{d}{d t} \eta(t)(p)\right|_{0}$ is an element of $T_{p}$. Hence the tangent space of $S$ at $I d$ is the set of functions $\{V\}$ from $M$ to $T$ such that $V(p) \in T_{p}$. This set is just $C^{\prime \prime}(T)$. For any element $V$ of $C^{\infty}(T)$ there is a unique one parameter group of diffeomorphisms $\eta(t)$ such that $\left.\frac{d}{d t}(\eta(t))\right|_{0}=V$. Hence to evaluate the tangent map of $\psi_{\prime \prime}$ at $V$, it is enough to know $\frac{d}{d t}((\eta(t)) *(g))$. But $\frac{d}{d t}\left(\eta^{*}(t)(g)\right)$ is by definition $\mathscr{L}^{\prime} \cdot(g)$, the Lie derivative of $V$ with respect to $g$. Now using $g$ to identify $A^{1}$ and $T$ we find by (2.1) that this map is $2 \delta^{*}$.

To prove $C^{\infty}\left(S^{2}\right)=\delta^{-1}(0) \oplus \delta^{*}\left(C^{\cdot}\left(A^{1}\right)\right)$, we must first derive some results on linear partial differential operators.

\section{Differential operators with injective symbol}

Let $E$ be a vector bundle over $M$ with a riemannian structure, i.e., with an inner product on each of the fibres of $E$, and fix a volume element $\mu$ on $M$. Then $C^{\infty}(E)$ gets an inner product and we call its completion $H^{\prime \prime}(E)$. If $J^{s}(E)$ is the bundle ${ }^{2}$ of $s$-jets of $E$, there is a natural map $j_{s}: C^{\prime \prime}(E)$ $\rightarrow C^{*}\left(J^{*}(E)\right.$ ). Giving $J^{*}(E)$ a riemannian structure we get an inner product on $C^{\infty}\left(J^{s}(E)\right)$. We define \langle\rangle$_{s}$ to be the inner product on $C^{\prime \prime}(E)$ induced by $j_{s}$ and the inner product on $C^{\infty}\left(J^{s}(E)\right) . H^{*}(E)$ is defined to be the completion of $C^{\infty}(E)$ in $\langle,\rangle_{s}$.

Assume $F$ is another such vector bundle, and let $D: C^{\omega}(E) \rightarrow C^{\prime \prime}(F)$ be a differential operator of order $k$. Then $D$ extends to a continuous linear map $D_{s}: H^{s}(E) \rightarrow H^{s-k}(F)$. Also $D^{*}: C^{\infty}(F) \rightarrow C^{\prime \prime}(E)$ has order $k$, so it can be extended to a map $D_{s-k}^{*}: H^{s-k}(F) \rightarrow H^{s-2 k}(E)$. We recall that for any $p \in M$ and

\footnotetext{
${ }^{2}$ For a definition of $J^{s}(E), H^{s}(E)$, and details of this section, see [8].
} 
any cotangent vector $t \in T_{p}^{*}$, there is a linear map $\sigma_{t}(D): E_{p} \rightarrow F_{p}$ called the symbol of $D$. Also $\sigma_{t}\left(D^{*}\right): F_{p} \rightarrow E_{p}$ is the adjoint of $\sigma_{t}(D)$ with respect to the inner products on $E_{p}$ and $F_{p}$. We say that the symbol of $D$ is injective, if $\sigma_{t}(D)$ is injective for all non-zero $t$.

The goal of this section is to prove:

Theorem 4.1. ${ }^{3}$ If $D$ has injective symbol, then

$$
H^{s-k}(F)=D_{s}\left(H^{s}(E)\right) \oplus\left(D_{s-k}^{*}\right)^{-1}(0),
$$

and the summands are orthogonal with respect to the inner product $\langle,>$ of $H^{0}(F)$.

Corollary 4.2. $C^{\infty}(F)=D\left(C^{\infty}(E)\right) \oplus\left(D^{*}\right)^{-1}(0)$, the summands being orthogonal as above.

To prove the above theorem we will need the notion of an elliptic operator: we say an operator $\alpha: C^{\infty}(E) \rightarrow C^{\infty}(F)$ of order $k$ is elliptic if $\sigma_{\iota}(\alpha): E_{p} \rightarrow F_{p}$ is an isomorphism for all non-zero $t$. A fundamental property of elliptic operators is:

Regularity Theorem 4.3. If $\alpha$ is elliptic of order $k$, then

$$
H^{s-k}(F)=\alpha_{s}\left(H^{s}(E)\right) \oplus \operatorname{ker} \alpha_{s-k}^{*},
$$

and $C^{\infty}(F)=\alpha\left(C^{\infty}(E)\right) \oplus \operatorname{ker} \alpha^{*}$. Also if for $l>s-k, \alpha_{s}(x) \in H^{l}(F)$, then $x \in H^{l+k}(E)$, and if $\alpha_{s}(x) \in C^{\infty}(F)$, then $x \in C^{\infty}(E)$.

Proof. [8, Chapter XI].

Also we need two lemmas.

Lemma 4.4. If $D$ is an operator of order $k$ with injective symbol, then $D^{*} D$ is an elliptic operator of order $2 k$.

Proof. $\sigma_{t}\left(D^{*} D\right)=\sigma_{t}\left(D^{*}\right) \sigma_{t}(D)=\left(\sigma_{t}(D)\right)^{*} \sigma_{t}(D)$, where the "** on the right hand side means the adjoint of the operator $\sigma_{t}(D): E_{p} \rightarrow F_{p} . \sigma_{t}(D)$ injective implies $\left(\sigma_{t}(D)\right)^{*} \sigma_{t}(D)$ is an isomorphism.

Lemma 4.5. Let $X$ and $Y$ be Banach spaces, and $T: X \rightarrow Y$ a bounded linear map. Assume $C$, a closed subspace of $Y$, is an algebraic linear complement to $T(X)$. Then $T(X)$ is closed in $Y$, and $Y=T(X) \oplus C$ topologically.

Proof. See [8, Proof of Theorem 1, p. 119].

Now we are ready to prove our theorem.

We first show that $\left(D_{s-k} D_{s}^{*}\right)^{-1}(0)=\left(D_{s}^{*}\right)^{-1}(0)$ and $\left(D^{*} D\right)^{-1}(0)=D^{-1}(0)$. Clearly $\left(D_{s-k} D_{s}^{*}\right)^{-1}(0) \supseteq\left(D_{t}^{*}\right)^{-1}(0)$. If $D_{s-k} D_{s}^{*}(x)=0$, then $\left\langle x, D_{s-k} D_{s}^{*} x\right\rangle$ $=\left\langle D_{s}^{*} x, D_{s}^{*} x\right\rangle=0$ so $D_{s}^{*}(x)=0$. Similarly $\left(D^{*} D\right)^{-1}(0) \supseteq D^{-1}(0)$, and $D^{*} D x$ $=0$ implies $\left\langle x, D^{*} D x\right\rangle=\langle D x, D x\rangle=0$ so $D x=0$.

Secondly we show $D_{s}\left(H^{s}(E)\right)=D_{s} D_{s+k}^{*}\left(H^{s-k}(F)\right)$. Clearly $D_{s}\left(H^{s}(E)\right)$ $\supseteq D_{s} D_{s+k}^{*}\left(H^{s+k}(F)\right)$. If $x=D_{s} y, y \in H^{s}(E)$, then $y=D_{s+k}^{*} D_{s}(a)+b$ by the

${ }^{3}$ This theorem is a special case of the statement involving equation (13) on page 447 of [13]. Since the proof of much easier in the special case, we include it. 
regularity theorem, where $a \in H^{s+2 k}(E)$ and $b \in\left(D^{*} D\right)^{-1}(0)$. But then by the above $b \in D^{-1}(0)$ so

$$
x=D_{s}(y)=D_{s} D_{s+k}^{*} D_{s+2 k}(a) .
$$

By Lemma 4.5, in order to prove our theorem, we need only show $H^{s}(F)=D_{s}\left(H^{s}(E)\right) \oplus\left(D_{s-k}^{*}\right)^{-1}(0)$ algebraically, since $\left(D_{s-k}^{*}\right)^{-1}(0)$ is closed. $\left(D_{s-k}^{*}\right)^{-1}(0) \cap D_{s}\left(H^{s}(E)\right)=\{0\}$ because if $D_{s-k}^{*} D_{s} x=0$ then $\left\langle x, D_{s-k}^{*} D_{s} x\right\rangle$ $=\left\langle D_{s} x, D_{s} x\right\rangle=0$, so $D_{s}(x)=0$.

Also

$$
\begin{aligned}
H^{s-k}(F) & =\left(D_{s-2 k} D_{s-k}^{*}\right)^{-1}\left(D_{s-2 k} D_{s-k}^{*}\right)\left(H^{s-k}(F)\right) \\
& =\left(D_{s-2 k} D_{s-k}^{*}\right)^{-1}\left(D_{s-2 k}\left(H^{s-2 k}(E)\right)\right) \\
& =D_{s-k}\left(H^{s-k}(E)\right) \cap H^{s-k}(F)+\left(D_{s-2 k} D_{s-k}^{*}\right)^{-1}(0) .
\end{aligned}
$$

But $D_{s-2 k}(x) \in H^{s-k}(F)$ implies $D_{s-3 k}^{*} D_{s-2 k}(x) \in H^{s-2 k}(E)$, which by regularity implies that $x \in H^{s}(E)$. Also $\left(D_{s-2 k} D_{s-k}^{*}\right)^{-1}(0)=\left(D_{s-k}^{*}\right)^{-1}(0)$ as we have shown above, so $H^{s-k}(F)=D_{s}\left(H^{s}(E)\right) \oplus\left(D_{s-k}^{*}\right)^{-1}(0)$. The summands are orthogonal by the fact that $\left\langle D_{s} x, y\right\rangle=\left\langle y, D_{s}^{*} x\right\rangle$.

Proof of Corollary. First we show that $C^{\infty}(F)=D\left(C^{\infty}(E)\right) \oplus D^{-1}(0)$ algebraically. From the theorem we know that the two summands have zero intersection, so we need only show that they span. Given $f \in C^{\cdots}(F)$ we know $f=D_{s}(e)+h, e \in H^{*}(E), h \in\left(D_{s-k}^{*}\right)^{-1}(0)$. Therefore $D^{*} f=D_{s-k}^{*} D_{s}(e)$. But $D^{*} f \in C^{\infty}(E)$, so by regularity $e \in C^{\prime \prime}(E)$ and hence $h=f-D(e) \in C^{\infty}(F)$.

Now we show the sum is topological. In the proof of the theorem we showed that

$$
D\left(H^{*}(E)\right) \cap H^{t}(F)=D\left(H^{t+k}(E)\right)
$$

for any $t \geq s-k$. But $C^{\infty}(F)=\bigcap_{t \geq s} H^{t}(F)$, so $D\left(C^{\infty}(E)\right)=C^{\infty}(F) \cap D_{s}\left(H^{s}(E)\right)$, and $D\left(C^{\infty}(E)\right)$ is closed in $C^{\infty}(F)$. The other summand is also closed, so the sum must be topological.

Now to prove $C^{\infty}\left(S^{2}\right)=\delta^{*}\left(C^{\infty}\left(A^{\prime}\right)\right) \oplus \delta^{-1}(0)$, we need only look at the symbol of $\delta^{*}$. A direct calculation shows $\sigma_{t}\left(\delta^{*}\right)(\xi)=\frac{1}{2}(\xi \oplus t+t \oplus \xi)$, which is clearly injective for non-zero $t$.

\section{Deformations of scalar curvature}

Another splitting of $C^{\infty}\left(S^{2}\right)$ was kindly communicated to us by $\mathrm{L}$. Nirenberg and is based on a conversation between him and L. Fadeev. To define it we need the following two operators: $\alpha: A^{1} \rightarrow S^{2}$, defined by $\alpha(\xi)=\delta^{*} \xi+(\delta \xi) g$, and $\beta: A^{0} \rightarrow S^{2}$, defined by $\beta(f)=\operatorname{Hess}(f)-f \rho+\left(\frac{1}{n-1}\right) f \tau g$. 
$\sigma_{t}(\delta)(\xi)=(t, \xi)$, so $\sigma_{t}(\alpha)(\xi)=\frac{1}{2}(t \otimes \xi+\xi \otimes t)+(t, \xi) g$. It is easy to check that this is injective. $\beta$ is a second order operator, and $\sigma_{t}(\beta)(f)$ $=\sigma_{t}($ Hess $)(f)=f(t \otimes t)$, which is also injective. Hence $C^{\infty}\left(S^{2}\right)=\alpha\left(C^{\infty}\left(A^{1}\right)\right)$ $\oplus \alpha^{-1}(0)$ and $C^{\infty}\left(S^{2}\right)=\beta\left(C^{\infty}\left(A^{1}\right)\right) \oplus \beta^{-1}(0)$.

Lemma 5.1. If $(M, g)$ has constant scalar curvature $\tau$, then $\alpha\left(A^{1}\right)$ and $\beta\left(A^{0}\right)$ are orthogonal with respect to the standard inner product $\langle,>$ on $C^{\infty}\left(S^{2} T^{*}\right)$.

Theorem 5.2. If $(M, g)$ has constant scallar curvature, then

$$
C^{\infty}\left(S^{2}\right)=\alpha\left(C^{\infty}\left(A^{1}\right)\right) \oplus \beta\left(C^{\infty}\left(A^{0}\right)\right) \oplus \alpha^{*-1}(0) \cap \beta^{*-1}(0),
$$

where the summands are orthogonl.

Proof. The theorem follows immediately from the lemma and the two decompositions of $C^{\infty}\left(S^{2}\right)$ above.

To prove Lemma 5.1 we must introduce a second order operator $\gamma: S^{2} \rightarrow A^{0}$. $\gamma$ is defined as follows: given $h \in S^{2}$, let $g(t)$ be a curve in $\mathscr{M}$ such that $g(0)=g$ and $\left.\frac{d}{d t} g(t)\right|_{0}=h$. Let $\tau(t)$ be the scalar curvature of the metric $g(t)$ and define $\gamma(h)$ to be $\left.\frac{d}{d t} \tau(t)\right|_{0}$. From [2, (3.4)] we find

$$
\gamma(h)=\Delta \operatorname{tr}(h)+\delta \delta(h)-(h, \rho) .
$$

Since $\delta^{*}(\xi)=\frac{1}{2} \mathscr{L}_{\xi^{\prime}}(g)$ for $\xi \in A^{1}$, it is clear that $\gamma \circ \delta(\xi)=\frac{1}{2} \mathscr{L}_{\xi^{\prime}}(\tau)$, so in particular if $\tau$ is constant $\gamma \circ \delta^{*}=0$.

It is easy to check that the symbol of $\gamma$ is surjective, so $\sigma_{t}\left(\gamma^{*}\right)$ must be injective. Hence we get an orthogonal splitting $C^{\infty}\left(S^{2}\right)=\gamma^{*}\left(A^{0}\right) \oplus \gamma^{-1}(0)$. Since $\gamma \circ \delta^{*}=0, \gamma^{*}\left(A^{0}\right)$ and $\delta^{*}\left(A^{1}\right)$ orthogonal, so that we can get a finer splitting:

$$
S^{2}=\gamma^{*}\left(A^{0}\right) \oplus \delta^{*}\left(A^{\prime}\right) \oplus \delta^{-1}(0) \cap \gamma^{-1}(0)
$$

This is very similar to the splitting of Theorem 5.2, to which we now return. We prove Lemma 5.1 .

We need only show $\beta\left(A^{0}\right) \subseteq \alpha^{*-1}(0)$, for $\alpha^{*-1}(0)$ is orthogonal to $\alpha\left(A^{\prime}\right)$. First we remark that from (5.3) we easily get

$$
\gamma^{*}(f)=(\Delta f) g-f \rho+\delta^{*} d f
$$

Also since $\gamma \circ \delta^{*}=0, \delta \circ \gamma^{*}=0$ so that

$$
\delta((\Delta f) g)-\delta(f \rho)+\delta \delta^{*} d f=0 .
$$

To prove the lemma we must show $\alpha^{*} \circ \beta=0$, and from the formulas for $\alpha$ and $\beta$, 


$$
\begin{aligned}
\alpha^{*} \circ \beta(f)= & (\delta+d \circ \operatorname{tr})\left(\operatorname{Hess}(f)-f \rho+\frac{1}{n-1} f \tau g\right) \\
= & \delta \delta^{*} d(f)-\delta(\rho f)+\left(\frac{1}{n-1}\right) \tau \delta(f g)-d \Delta f \\
& -\tau d f+\left(\frac{1}{n-1}\right) \tau d f
\end{aligned}
$$

But,

$$
\delta(f g)=-d f,
$$

so

$$
\alpha^{*} \circ \beta(f)=\delta \delta^{*} d(f)-\delta(\rho f)-d \Delta f+\left(\frac{-1}{n-1}-1+\frac{n}{n-1}\right) \tau d f .
$$

Using (5.6) and (5.8), we find that $\delta \delta^{*} d(f)-\delta(\rho f)-d \Delta f=0$, and the last term on the right hand side is also zero, so the lemma is proved.

Remark 5.10. S. Deser discusses decompositions like $(3.1),(5.2)$ and (5.4) in some articles on general relativity for the purpose of finding a canonical form for the gravitational field. See [11, pp. 158-162] and [12].

Remark 5.11. C. Barbance [1] proved our original splitting $S^{\prime \prime}=\delta^{\prime}(0)$ $+\delta^{*}\left(A^{1}\right)$ in the case where $M$ is an Einstein manifold. She also gave a more refined splitting for this case.

Remark 5.12. E. Calabi [3] has investigated the operator $\delta^{*}: A^{1} \rightarrow S^{\prime \prime}$ in the case where $M$ has constant curvature. He formed a sequence of differential operators

$$
\left(\delta^{*}\right)^{-1}(0) \rightarrow A^{1} \stackrel{\delta^{*}}{\longrightarrow} S^{2} \stackrel{D_{1}}{\longrightarrow} S^{\prime}\left(A^{2}\right) \stackrel{D_{2}}{\longrightarrow} B_{3} \stackrel{D_{3}}{\longrightarrow} \ldots \stackrel{D_{n !}}{\longrightarrow} B_{\|}^{\longrightarrow} 0,
$$

which is locally exact and resolves the sheaf defined by $\left(i^{*}\right)^{-1}(0) . D_{1}$ is defined just as $\gamma$ is, except that the full curvature tensor replaces the scalar curvature. $S^{2}\left(A^{2}\right)$ means the symmetric tensor of $A^{2}$ with itself, and the $\left\{B_{i}\right\}$ are certain subbundles of $A^{2} \otimes A^{i}$.

\section{Four elliptic operators on $S^{2}$}

a. The rough laplacian $\bar{J}$. For any $r, s$ it is easy to check the covariant derivative $\nabla: T_{s}^{r} \rightarrow T_{s+1}^{r}$, has injective symbol. Hence by Lemma 4.4 the operator $\bar{\Delta}=\nabla^{*} \nabla=\delta \nabla: T_{s}^{r} \rightarrow T_{s}^{r}$ is an elliptic operator on $T_{s}^{r}$, called the rough laplacian. In local coordinates:

$$
(\bar{J} t)_{i_{1} \ldots i_{s}}^{j_{1} \ldots j_{r}}=-\sum_{l} \Gamma^{\prime} \Gamma_{1} t_{i_{1} \ldots i_{s}}^{j_{1} \ldots i_{r}}
$$


Moreover $\bar{\Delta}$ is non-negative : $\langle\bar{\Delta} t, t\rangle=\|\Delta t\|^{2} \geq 0$, and so $\operatorname{ker} \bar{\Delta}=\operatorname{ker} \Delta$. For $T_{0}^{0}=A^{0}$ the rough laplacian $\bar{\Delta}$ is nothing but $\Delta$, the usual Laplace-Beltrami operator for functions on $M$.

Note also that $\bar{\Delta} S^{2} \subset S^{2}$, so we can consider $\bar{\Delta}: S^{2} \rightarrow S^{2}$ as an operator on $S^{2}$ (using the same notation $\bar{\Delta}$ ). From $\nabla g=0$ :

$$
\bar{\Delta} g=0 \text {. }
$$

It is also elementary that:

$$
\operatorname{ker} \nabla=\boldsymbol{R} \cdot g \text { iff }(M, g) \text { locally irreducible . }
$$

Using the trace $\operatorname{tr}: S^{2} \rightarrow A^{0}$, from (6.1) one deduces:

$$
\operatorname{tr} \cdot \bar{\Delta}=\Delta \circ \operatorname{tr} .
$$

$A$ s a consequence of (6.2), we can consider $\bar{J}$ as an operator $\bar{d}: T Z \rightarrow T Z$.

b. The operator $\Theta$. Define $\square: S^{2} \rightarrow S^{2} \otimes A^{1}$ by

$$
(\square h)(x, y ; z)=\nabla_{z} h(x, y)-\nabla_{x} h(z, y)-\nabla_{y} h(z, x),
$$

and consider $(\square, \sqrt{ } 2 \delta): S^{2} \rightarrow\left(S^{2} \otimes A^{1}\right) \times A^{1}$. It is straightforward to check this operator has injective symbol. Hence if we set $\Theta=(\square, \sqrt{ } 2 \delta)^{*}([], \sqrt{ } 2 \delta)=$ $\square * \square+2 \delta^{*} \delta$, the so-gotten operator $\Theta$ is elliptic non-negative (Lemma 4.4): $\langle\Theta h, h)\rangle=\|\left[h\left\|^{2}+2\right\| \delta h \|^{2} \geq 0\right.$ and $\operatorname{ker} \Theta=\operatorname{ker} \square \cap \operatorname{ker} \delta$. In fact : $\operatorname{ker} \square=$ $\operatorname{ker} \nabla=\operatorname{ker} \Delta$; for by (6.3), $\square h=0$ implies that $\nabla h$ is antisymmetric in the two last entries, and being symmetric in the two first entries it has to be zero.

To get an explicit formula for $\Theta$, we perform explicit computations (straightforward ones with the Ricci commutation formulas) in local coordinates:

$$
\left(\square^{*} k\right)_{a b}=\sum_{l}\left(\nabla^{\prime} k_{l a b}+\nabla^{\prime} k_{l b a}-\nabla^{\prime} k_{a b l}\right) .
$$

Therefore

$$
\Theta=3 \mathrm{~J}+K \text {, }
$$

where $K: S^{2} \rightarrow S^{2}$ is the zero-order differential operator defined explicitly by

$$
(K h)_{a b}=\sum_{l}\left(\rho_{a l} h_{b}^{l}+\rho_{b l} h_{a}^{l}\right)-2 \sum_{l, m} R_{a l b m} h^{l m} .
$$

Direct verification yields

$$
\begin{gathered}
K \cdot g=0, \text { so } \theta \cdot g=0, \\
\operatorname{tr} \circ K=0 .
\end{gathered}
$$

Hence by (6.2), 


$$
\operatorname{tr} \circ \Theta=\Delta \circ \operatorname{tr}
$$

so that $\Theta$ also preserves $T Z$ and can be considered as an operator $\theta: T Z \rightarrow T Z$. On $T Z$ the operator $K$ is intimately related to the sectional curvature of $(M, g)$ as follows :

Proposition 6.1. The operator $K$ is positive definite on $T Z$ if $(M, g)$ is of strictly positive sectional curvature.

Proof. At any point $m \in M$, diagonalize $h$ with respect to $g$, using an orthonormal basis $\left\{e_{a}\right\}$. Letting the sectional curvature of the plane of $T_{m}(M)$ generated by $e_{a}$ and $e_{b}$ be $\sigma\left(e_{a}, e_{b}\right)$ and expressing $\sigma\left(e_{a}, e_{b}\right)$ in terms of $R$, by a direct computation from (6.5) we get

$$
(K h \mid h)=\sum_{a \neq b} \sigma\left(e_{a}, e_{b}\right)\left(h_{a u}-h_{b u}\right)^{2} .
$$

Hence $(K h \mid h) \geq 0$, and $(K h \mid h)=0$ implies that all the $h_{a a}$ 's are equal; but $\operatorname{tr} h=\sum_{a} h_{a u}$, so all the $h_{a a}$ 's have to be zero.

c. The operator $\Psi$. This operator is the one associated to a differential system introduced by J. Simons in [9, pp. 96-97]. Define $\sigma: S^{2} \rightarrow A^{2} \otimes A^{1}$ by $(\sigma h)(x, y ; z)=\nabla_{x} h(y, z)-\nabla_{y} h(x, z)$ and $(\sigma, \sqrt{ } 2 \delta): S^{2} \rightarrow\left(A^{2} \otimes A^{1}\right) \times A^{1}$. One can check it has injective symbol, so $\Psi=(\sigma, \sqrt{ } 2 \delta)^{*}(\sigma, \sqrt{ } 2 \delta)=\sigma^{*} \sigma$ $+2 \delta^{*} \delta$ is elliptic, non-negative: $\langle\Psi h, h\rangle=\|\sigma h\|^{2}+2\|\delta h\|^{2} \geq 0$ and $\operatorname{ker} \Psi=$ $\operatorname{ker} \sigma \cap \operatorname{ker} \delta$. Direct computations yield

$$
\begin{aligned}
\left(\sigma^{*} k\right)_{a \iota} & =-\sum_{l}\left(\nabla^{\prime} k_{l u b}+\nabla^{\prime} k_{l b u}\right), \\
\Psi & =2 \bar{\jmath}+K .
\end{aligned}
$$

By (6.7) we still have $\operatorname{tr} \circ \Psi=\Delta \circ \operatorname{tr}$ and a restricted operator $\Psi: T Z \rightarrow T Z$.

In [9] J. Simons introduced the system $\delta h=\sigma h=0$, because if $(M, g)$ $\subset\left(S^{n+1}, g_{0}\right)$ is a minimal hypersurface of the standard sphere $\left(S^{n+1}, g_{0}\right)$, then its second fundamental form $h$ satisfies $\delta h=\sigma h=0$ (in fact $\sigma h=0$ and $\operatorname{tr} h$ $=0$, which implies immediately $\sigma h=0$ ). Combining this with Proportion 6.1, we get:

Corollary 6.2. If $(M, g) \subset\left(S^{n+1}, g_{0}\right)$ is a compact minimal hypersurface, and $(M, g)$ is of strictly positive sectional curvature, then $(M, g)$ has to be an equator of $\left(S^{n+1}, g_{0}\right)$.

Note this is a best possible result, since the flat standard square two-dimensional torus has a minimal imbedding in $\left(S^{3}, g_{0}\right)$.

d. The operator $\Delta$. This operator was introduced by Lichnerowicz in [6, p. 27]. It is defined in an explicit way as $\Delta=\bar{d}+K$. If we write out $\Delta$ in local coordinates, the formula has the property that, when one replaces $h \in S^{2}$ by $\alpha \in A^{2}$ then $\Delta h$ becomes $\Delta \alpha$, the usual laplacian of de Rham on exterior forms. We do not known if $\Delta$, which is of course elliptic, is still non-negative. We again have $\operatorname{tr} \circ \Delta=\Delta \circ \operatorname{tr}$ (see [6, last half of p. 27]). 
e. Orthogonal decompositions. By the general theorem on elliptic operators (4.3), we have the orthogonal decompositions: $S^{2}=\operatorname{ker} \nabla \oplus \bar{\Delta}\left(S^{2}\right), S^{2}=$ $\operatorname{ker} \nabla \oplus \Theta\left(S^{2}\right), S^{2}=\operatorname{ker} \Psi \oplus \Psi\left(S^{2}\right), S^{2}=\operatorname{ker} \Delta \oplus \Delta\left(S^{2}\right)$. But, contrary to the case of the laplacian on exterior forms, the sums $\Theta\left(S^{2}\right)=\delta^{*} A^{1}+\square *\left(S^{2} \otimes A^{1}\right)$, $\Psi\left(S^{2}\right)=\delta^{*} A^{1}+\sigma^{*}\left(A^{2} \otimes A^{1}\right)$ are neither direct nor orthogonal, for $\delta \circ \sigma^{*} \neq 0$ and $\delta \circ \square^{k} \neq 0$.

\section{Deformations of Einstein manifolds}

An Einstein manifold is a riemannian manifold $(M, g)$ for which the Ricci curvature satisfies $\rho=k \cdot g$ (for some real number $k$ ).

By a deformation of Einstein structures through $g$ we mean a smooth curve $g(t)$ ( $t$ running through some open interval containing 0 ) in $\mathscr{H}$ with $g(0)=g$ and such that, for all $t$, there exists $k(t)$, a real number, with $\rho_{g(t)}=k(t) \cdot g(t)$. If $k(0) \neq 0$, by normalization we can replace our deformation by another one such that $\rho_{g(t)}=\varepsilon \cdot g(t), \varepsilon= \pm 1$. In the following we assume this is done. In the case $k(0)=0$, we do not know if such a deformation exists with $k(\mathrm{t})$ not identically 0 ; it is an interesting problem. We now compute consequences of the equation $\rho_{g(t)}=k(t) \cdot g(t)$. The formula giving $d \rho_{g}(t) / d t$ is classical; we write it down as in [2, Formula (3.3) for $t=0$ ], all invariants (like $\delta, \delta_{*}, \Delta$, Hess) being understood to be with respect to $g=g(0)$ :

$$
\frac{d \rho_{g(t)}}{d t}(0)=\frac{1}{2}\left(\Delta h+2 \delta^{*} \delta h-\operatorname{Hess}(\operatorname{tr} h)\right), h=\underset{d t}{d g(t)}(0)
$$

From $\S 3$, we know we can assume $\delta h=0$, so

$$
\frac{d \rho_{g(t)}}{d t}(0)=\frac{1}{2}(\Delta h-\text { Hess }(\operatorname{tr} h))
$$

Lemma 7.1. The function $\operatorname{tr} h$ is necessarily constant.

The proof depends on whether $k(0)$ is $1,-1$, or 0 .

a. $k(0)=0$. By hypothesis we have $\Delta h-$ Hess $(\operatorname{tr} h)=2 k^{\prime}(0) \cdot g+2 k(0) h$ $=2 k^{\prime}(0) \cdot g$. Taking the trace of both sides and using $6 d$ and $\Delta=-\operatorname{tr} \circ$ Hess we get $\Delta(\operatorname{tr} h)=n k^{\prime}(0)$ which implies $k^{\prime}(0)=0$ and $\operatorname{tr} h$ is constant.

b. $k(0)=-1$. In this case, $\rho_{g(t)}=-g(t)$ for all $t$ so that taking traces again: $\Delta(\operatorname{tr} h)=-\operatorname{tr} h$, which implies $\operatorname{tr} h=0$.

c. $k(0)=1$. Now we have $\Delta(\operatorname{tr} h)=\operatorname{tr} h$. We apply [5, p. 135] to the effect that $\lambda_{1}$, the first eigenvalue of $\Delta$ on $A^{0}$, is $\geq \frac{n}{n-1} \cdot \alpha$, where $\alpha$ is a lower bound for $\rho$, i.e., $\rho \geq \alpha \cdot g$. But here one can take $\alpha=1$, so $\lambda_{1} \geq$ $\frac{n}{n-1}>1$, and hence $\operatorname{tr} h=0$. 
From the lemma we nave now the standard equation for $h=\frac{d g(t)}{d t}(0)$ of a deformation of Einstein structures:

$$
\Delta h=\varepsilon \cdot h, \quad \varepsilon=1,-1,0: \rho=\varepsilon \cdot g .
$$

Since $\Delta$ is elliptic, it has finite dimensional kernel, so from $\S 3$ we get

Corollary 7.2. The subset $\pi(\xi)$ of Einstein structures in $\| / D$ is finite dimensional.

Remark 1. We can define a solution of (7.1) as an infinitesimal deformation of Einstein structures.

Remark 2. We proved, precisely, that the tangent direction $h$ has to belong to $\operatorname{ker}(\Delta-\varepsilon)$. The converse might be true: if $h \in \operatorname{ker}(\Delta-\varepsilon)$, then there exists a deformation of Einstein structures with $h$ as tangent direction. This existence theorem extending an infinitesimal deformation into a local one seems to be out of the reach of the present results of non-linear analysis.

Using the definition $\Delta=\vec{\Delta}+K$, the definition of $K$ in (6.5), and the fact that $\rho=\varepsilon \cdot g$ we can write (7.1) more explicitly:

$$
\Delta-\varepsilon=\bar{\Delta}+L,
$$

where $L$ is defined explicitly by

$$
(L h)_{a b}=-\sum_{l, m} R_{a l b m} h^{l m}
$$

In the case $\varepsilon=-1$ or $\varepsilon=0$ there exist deformations of Einstein structures, for example the flat riemannian structures on tori, or the families of constant negative curvature on a surface of genus greater than one. In the case $\varepsilon=+1$, we have, by the above:

Corollary 7.3. If $L$ is positive-definite on $T Z$, then $(M, g)$ is not infinitesimally deformable, i.e., $\operatorname{dim}_{g} \mathscr{E}=0$.

This happens, for example, in the following:

Lemma 7.4. If the sectional curvature of $(M, g)$ ranges in the interval ]$\left.\frac{p-1}{p}, 1\right]$ for $n=2 p$ or in the interval $\left.] \frac{2 p^{2}-1}{2 p(p+1)}, 1\right]$ for $n=2 p+1$, then $L$ is positive-definite on $T Z$.

Proof. This lemma is very elementary. At a given point, we diagonalize $h$ with respect to $g$, using an orthonormal basis $\left\langle e_{a}\right\rangle$; set $h_{a a}=x_{a}, \sigma\left(e_{a}, e_{b}\right)=\alpha_{a b}$. Then

$$
\begin{aligned}
\frac{1}{2}(L h \mid h) & =-\sum_{a \neq b} \alpha_{a b} x_{a} x_{b}=\left(\sum_{a} x_{a}\right)^{2}-\sum_{a \neq b} \alpha_{a b} x_{a} x_{b} \\
& =\sum_{a} x_{a}^{2}+\sum_{a \neq b}\left(1-\alpha_{a b}\right) x_{a} x_{b} .
\end{aligned}
$$


Separate the $x_{a}$ 's in $\geq 0$ and $\leq 0: y_{i} \geq 0(i=1, \cdots, p), z_{j} \leq 0(j=1, \cdots, q)$ and set $A=\sum_{i} y_{i}=-\left(\sum_{j} z_{j}\right)$. We have also $1-\alpha_{a b} \geq \varepsilon \geq 0, \varepsilon$ to be found later. But

$$
\begin{aligned}
\frac{1}{2}(L h \mid h) & \geq \sum_{i} y_{i}^{2}+\sum_{j} z_{j}^{2}+\sum_{i, j} \varepsilon y_{i} z_{j} \\
& =\sum_{i} y_{i}^{2}+\sum_{j} z_{j}^{2}+2 \varepsilon\left(\sum_{i} y_{i}\right)\left(\sum_{j} z_{j}\right) \geq \frac{A^{2}}{p}+\frac{A^{2}}{q}-2 \varepsilon A^{2},
\end{aligned}
$$

which is positive if $\varepsilon \leq \frac{1}{2}\left(\frac{1}{p}+\frac{1}{q}\right)$. Taking the minimum value for this, i.e., $p=q=\frac{n}{2}$ if $n=2 p$, and $q=p+1$ if $n=2 p+1$, the lemma is proved.

Remark. In the interval $\left.] \frac{n-2}{n-1}, 1\right]$, see [2, Proposition (6.4)].

\section{Corrections and addition to [2]}

We wish to take the opportunity, writing on Einstein manifolds, to correct some mistakes made by Berger in [2].

a. In formula (3.2), p. 38 , read at the end a " + ". In formula (3.3) put a "_" in front of $\mathscr{D} d h$, in (3.4) "+" in front of $\delta \delta h$.

b. The number $i(\gamma)$ defined on the last line of p. 39 makes sense only when $n / 2$, and so does everything which follows in $\S 4$ of [2].

c. The end of p. 40 is incorrect, in the sense one should add everywherc the condition " $\tau$ is nowhere zero". In fact the best way to get Einstein structures as critical is to take $i(\gamma)=\int_{M} \tau_{r} \cdot v_{\gamma}$ under the normalization $\int_{M} \tau_{r}=1$.

d. The first remark of p. 54 (and so the Proposition (8.1)) is simply proved by Bochner's theorem [14, Theorem 2.9., p.37]. If $(M, g)$ is flat and compact, then it has $[10$, Theorem 3.3 .1$, p. 105$]$ a covering $(\bar{M}, \bar{g})$ which is a flat torus. But

Addition 8.1. On a torus, there are no Einstein structures with $\varepsilon=1$ (i.c., $\rho=g$ ), and any Einstein structure with $\rho=0$ is necessarily flat.

Proof. By the quoted result of Bochner, every harmonic 1-form has zero covariant derivative if $\rho=0$, and is zero if $\rho>0$. By the de Rham theorem and the Hodge-de Rham theorem there are $n(=\operatorname{dim} M=$ first Betti number of $M$ ) such linearly independent such harmonic 1-forms; hence our manifold carries $n$ linearly independent 1 -forms with zero covariant derivative, so it has to be flat.

Remark. It would be interesting to decide whether or not a torus can carry an Einstein structure with $\varepsilon=-1$, i.e., $\rho=-g$ (this question is a particular case of the problem which arose at the beginning of $\S 5$ ). 


\title{
Bibliography
}

[1] C. Barbance, Décomposition d'un tenseur symétrique sur un espace d'Einstein, C. R. Acad. Paris 258 (1964) 5336-5338, 264 (1967) 515-516.

[2] M. Berger, Sur les variétés d'Einstein compactes, Comptes Rendus III' réunion math. expression latine, Namur 1965, 35-55.

[ 3 ] E. Calabi, On compact Riemannian manifolds with constant curvature. I, Proc. Sympos. Pure Math. Vol. 3, Amer. Math. Soc., 1961, 155-180.

[4] D. Ebin, The manifold of Riemannian metrics, Proc. of the 1968 Summer Institute on Global Analysis, Amer. Math. Soc.

[5] A. Lichnerowicz Géométrie des groupes de transformations, Dunod, Paris, 1958.

[6] - Propagateurs et commutateurs en relativité generale, Inst. Hautes Études Sci. Publ. Math. No. 10 (1961) 293-344.

[ 7 ] H. Omori, On the group of diffeomorphisms of a compact manifold, Proc. of the 1968 Summer Institute on Global Analysis, Amer. Math. Soc.

[ 8 ] R. S. Palais, Seminar on the Atiyah-Singer index theorem. Ann. of Math. Studies, No. 57, Princeton University Press, Princeton, 1965.

[9] J. Simons, Minimal varieties in riemannian manifolds, Ann. of Math. 88 (1968) 62105.

[10] J. A. Wolf, Spaces of constant curvature, McCiraw-Hill, New York, 1967.

111] S. Deser, Covariant decomposition of a tensor and the gravitational Cauchy problem, Ann. Inst. H. Poincaré, sect. A, 7 (1967) 149-188.

[12] — Décomposition covariante é énergie du champ gravitationnel, Ann. Inst. H. Poincaré, sect. A, 8 (1968) 269-273.

[13] J. J. Kohn \& L. Nirenberg. Non-coercive houndary value problems, Comm. Pure Appl. Math. 18 (1965) 443-492.

[14] K. Yano \& S. Bochner, Curvature and Betti mumhers, Ann. of Math. Studies, No. 32. Princeton University Press, Princeton, 1953.

\author{
University of Paris \\ UNiVERSiTy OF CALIFORNIA, BERKEley
}

